\title{
25 Research Square \\ KRT17 Serves as an Oncogene and a Predictor of Poor Survival in Hepatocellular Carcinoma Patients
}

yuyan chen ( $\square$ chenyuyan95@163.com )

Affiliated Hospital of Nantong University https://orcid.org/0000-0002-8489-6961

Jing Chen

Affiliated Hospital of Nantong University

\section{Zu-Cheng Tian}

Haian Chinese Medical Hospital

\section{Dan-Hua Zhou}

Affiliated Hospital of Nantong University

Ran Ji

Affiliated Hospital of Nantong University

Si-Jia Ge

Affiliated Hospital of Nantong University

Zhao-Xiu Liu

Affiliated Hospital of Nantong University

Wei Huang

Affiliated Hospital of Nantong University

\section{Research}

Keywords: Hepatocellular carcinoma, Keratin 17, Immune infiltration Biomarker

Posted Date: December 3rd, 2020

DOI: https://doi.org/10.21203/rs.3.rs-117723/v1

License: (c) (i) This work is licensed under a Creative Commons Attribution 4.0 International License.

Read Full License 


\section{Abstract}

Background: Hepatocellular carcinoma (HCC) is the second most common cancer-associated cause of death globally. It is thus vital that novel diagnostic and prognostic biomarkers associated with early-stage HCC be identified. While keratin 17 (KRT17) has previously been reported to be associated with certain cancer types, its relationship with HCC remains to be defined.

Methods区The expression of KRT17 in the TCGA LIHC database and in 44 pairs of HCC patient samples was assessed via qRT-PCR, western blotting, and immunohistochemical staining. The prognostic relevance of KRT17 was assessed using Kaplan-Meir curves, while important cancer- and KRT17-related biological processes were defined through gene set enrichment analysis (GSEA). The functional link between KRT17 expression and tumor cell proliferation/survival was assessed through flow cytometry, colony formation assay, CCK-8 assay, and subcutaneous tumor model approaches. Protein-protein interaction (PPI) networks and analyses of immune cell infiltration were also employed to define key signaling pathways associated with KRT17 expression in HCC.

Results囚HCC tissue samples exhibited increased KRT17 mRNA and protein expression that was predictive of poorer patient survival $(P<0.001)$. GSEA and functional experiments revealed that KRT17 functioned as a regulator of $\mathrm{HCC}$ tumor cell survival, proliferation, and cell cycle progression in vitro and in vivo. PPI network analyses also revealed that KRT17 expression was linked to immune cell infiltration and activation in patients with HCC.

Conclusion: We found that increased KRT17 levels were associated with poorer survival, more aggressive disease, and altered immune cell infiltration in patients suffering from HCC. As such, KRT17 may function as an oncogene and a prognostic biomarker in this cancer type.

\section{Introduction}

Hepatocellular carcinoma (HCC) is a malignancy responsible for approximately 780,000 deaths each year, ranking as the second leading cancer-associated cause of death globally [1]. While hepatectomy can be used to remove tumors from HCC patients, overall prognosis remains poor with approximately $65 \%$ of patients ultimately succumbing to disease even after undergoing this form of treatment [2]. It is thus vital that the molecular mechanisms governing HCC progression be better understood in order to guide targeted treatment efforts for this deadly disease.

Keratin is an intermediate protein (IP) family member that plays vital structural and protective roles [3, 4]. Cysteine found in keratin can protect the liver against cirrhosis, fatty liver disease, and other related conditions [5]. Nam-On Ku et al. further determined that keratin was able to prevent the necrotic and apoptotic death of liver cells [6]. During the process of apoptosis, keratin 18 (KRT18) and KRT19 undergo caspase-mediated cleavage at conserved aspartate residues. Keratins are also important in the context of oncogenesis, with elevated expression of KRT17 that was associated with poor prognosis having been observed in the context of colorectal cancer, pancreatic cancer, and non-small cell lung cancer $[7,8]$. Prior 
evidence suggests that KRT17 can influence tumor proliferation and metastasis [9-13], but its function in HCC remains to be defined.

Herein, KRT17 expression levels and prognostic relevance were therefore assessed using extant transcriptomic data and samples collected from $44 \mathrm{HCC}$ patients at the Affiliated Hospital of Nantong University. Through functional analyses, we found that KRT17 is associated with poor HCC patient prognosis, indicating that it may represent a viable target for the treatment of this form of cancer.

\section{Materials}

\subsection{Patients and HCC tissues}

In total, 44 pairs of HCC tumor and paracancerous normal tissue samples were collected from patients at the Affiliated Hospital of Nantong University from 2012-2016. Patients included in this study were those that met the following criteria: (1) patients with HCC that had been diagnosed through alpha-fetoprotein serology, imaging studies, and pathological findings as per the guidelines of the American Association for the Study of Liver Diseases (AASLD) [14]; (2) patients who were positive for chronic viral hepatitis B infection as defined by t hepatitis B surface antigen positivity (+); (3) patients that had not undergone any prior liver resection of cancer treatment; (4) patients without any tumor invasion of the trunk or branches of the hepatic or portal veins as established via magnetic resonance imaging (MRI); and (5) patients free of other diffuse liver diseases such as primary biliary cirrhosis or primary sclerosing cholangitis. The Human Research Ethics Committee of Nantong University Affiliated Hospital approved this study, with all patients having provided written informed consent to participate.

\section{2 qRT-PCR}

RNA was isolated from tumor tissue and paracancerous tissue samples and reversed transcribed to yield cDNA which was used for qRT-PCR analyses. KRT17 primers used in this study were as follows: forward:5囚-GGTGGGTGGTGAGATCAATGT-3》 and reverse:5囚-CGCGGTTCAGTTCCTCTGTC ; GAPDH

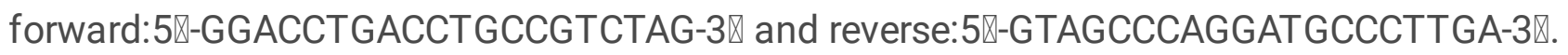

\subsection{Western blotting}

Western blotting was performed as in prior studies [15], with anti-GAPDH (Santa Cruz Biotechnology) being used as a loading control. Rabbit polyclonal anti-KRT17 (1:1000, \# 12509S, Cell Signaling Technology, Danvers, MA) was used to stain tumor and paracancerous tissue sections from HCC patients.

\subsection{Immunohistochemical (IHC) staining}

Rabbit polyclonal anti-KRT17 (1:100, \# 12509S, Cell Signaling Technology) was used to stain tumor and paracancerous tissue sections from HCC patients. Two experienced pathologists then independently scored KRT17 staining intensity (0: no staining, 1: weak positivity, 2: moderate positivity, 3: strong positivity) and the percentage of positively stained tumor cells (0: $0 \%, 1: 1-25 \%, 2: 26-50 \%, 3: 51-75 \%$, 
4: $76-100 \%)$. These two scores were then multiplied together to yield a final IHC score ranging from 0 to 12.

\subsection{TCGA LIHC database analyses}

TCGA LIHC RNA-Seq expression matrix and clinical data, including T stage and grade, were downloaded from the XENA tool (https://xenabrowser.net/heatmap/). The expression of KRT17 in these samples was then determined based upon $\log 2(x+1)$ values and transformed RSEM normalized counts.

\subsection{Gene set enrichment analysis (GSEA)}

Gene set enrichment between samples with high and low levels of KRT17 expression was defined using GSEA v.2.10.1, with both gene ontology (GO)[16] and KEGG[17] enrichment analyses being conducted. In total, 1000 gene set permutations were conducted per analysis.

\subsection{Protein-protein interaction (PPI) network construction}

A PPI network was constructed using the STRING (v10.0; http://string-db.org) database based upon key KRT17 co-regulated genes in order to assess potential interactions between proteins of interest [18]. The resultant network ultimately incorporated all putative interactions with an interaction score $>0.4$, and was visualized using Cytoscape (v.3.5) [19]. The ClueGO (v.2.5.3) and CluePedia (v.1.5.3) Cytoscape plugins, which enabled the visualization of non-redundant biological terms associated with large gene clusters in functional networks [20] [21], were used for GO and KEGG pathway-related analyses of co-expressed genes.

\subsection{TIMER analyses}

The tumor immune estimation resource (TIMER) tool was used to analyze tumor-infiltrating immune cells (https://cistrome.shinyapps.io/timer/)[22], estimating B cell, CD4 + T cell, CD8 + T cell, macrophage, dendritic cell, and neutrophil intratumoral infiltration based upon patterns of gene expression. This tool was used to compare patterns of immune cell infiltration as a function of KRT17 expression levels for samples in the LIHC database.

\subsection{Single-sample GSEA (sSGSEA) analyses}

The gsva R package was used to quantify immune cell infiltration via a sSGSEA approach [23] wherein gene signatures expressed by immune cell populations were applied to individual cancer samples.

\subsection{Cell lines and culture}

SMMC-7721, HepG2, Sk-Hep-G1, Huh7, HCC-LM3 cell lines, and THLE-2 cell lines were ordered from GeneChem (Shanghai, China). ALL liver cell lines were cultured in DMEM medium (Gibco, Gaithersburg, MD, USA) with $10 \%$ fetal bovine serum (Clark, Shanghai, China). The cell lines were authenticated by STR.

\subsection{Cell transfection}


KRT17 ectopic-expressing and empty vectors were obtained from GeneChem (Shanghai, China). Shorthairpin RNA (shRNA) sequences targeting KRT17 were procured from GenePharma (Soochow, China).

2.12 CCK-8, colony formation, cell cycle analysis, and apoptosis assays

These experiments were conducted as in a prior study [24].

\subsection{Mouse experiments}

Nude mice were subcutaneously implanted with $5 \times 10^{6}$ SMMC-7721/shKRT17 or control cells in the right axillary region. At 4 weeks post-tumor implantation, mice were sacrificed, and tumors were collected and imaged.

\subsection{Statistical analysis}

Expression profiles and clinical information of KRT17 and related genes in 374 HCC patients from TCGA and $44 \mathrm{HCC}$ patients from affiliated hospital of Nantong university were analyzed and displayed.

Statistical analyses were performed and visualized by using R version 4.0.2 and GraphPad Prism version 8.0 software. The quantitative values of all experiments were expressed as the mean \pm SD. The differences among/between sample groups were analyzed by independent samples t-test or one-way analysis of variance (ANOVA). Pearson's correlation coefficient was employed to measure the linear correlation between KRT17 and immune infiltrated cells, respectively. $\mathrm{P}<0.05$ was considered statistically significant.

\section{Results}

\subsection{Assessment of KRT17 expression and prognostic relevance in HCC}

In order to better understand KRT17 expression patterns in HCC patient tissues, we began by comparing these patterns using the TCGA LIHC database, revealing that KRT17 expression was elevated in HCC tissues $(n=372)$ relative to paracancerous normal tissues $(n=50, P<0.001$, Fig. $1 a)$. This finding was then confirmed via qRT-PCR, IHC, and Western blotting using 44 pairs of HCC and paracancerous tissue samples collected from the Affiliated Hospital of Nantong University (Fig. 1b-d). Kaplan-Meier survival curves revealed that the overall survival (OS) of patients with high levels of KRT17 expression was significantly reduced relative to that of patients with lower KRT17 expression levels $(P=0.048)$, and the TCGA LIHC dataset yielded comparable results $(P=0.016$, Fig. 1 e,f). One-way ANOVA analyses of the TCGA LIHC dataset further indicated that KRT17 expression levels were significantly associated with increased tumor T stage $(P=0.003)$ and grade $(P=0.037$, Fig. $1 \mathrm{~g}, \mathrm{~h})$. Together, these data revealed that KRT17 may function in an oncogenic manner and have value as a prognostic biomarker in patients with HCC. 


\subsection{The relationship between KRT17 expression levels and gene set enrichment analysis results}

Next, HCC patients in the TCGA LIHC database were stratified into KRT17-high and -low groups in order to explore the mechanistic basis for the putative oncogenic role of this keratin gene. GO and KEGG pathway functional enrichment analyses were conducted via a GSEA approach (Fig. 2a-d), revealing that KRT17 was associated with important functional pathways, including the regulation of intrinsic apoptotic signaling, the positive regulation of apoptotic signaling pathway, notch signaling pathway, methyl CpG binding, intestinal immune network for IGA production, and cell cycle signaling pathway (Fig. 2e-j).

\subsection{KRT17 knockdown modulates HCC cell proliferation and apoptosis, and promotes $\mathrm{G} 1$ phase arrest}

We next explored the functional role of KRT17 in HCC cell lines. Of seven tested HCC cell lines, expression of KRT17 was confirmed to be highest in SMMC-7721 cells and lowest in HCC-LM3 cells (Sup Fig. 1a). The apoptotic death and cell cycle progression of these cells was then assessed via flow cytometry, revealing higher numbers of cells in the G1 phase in the SMMC-7721-shKRT17 group relative to the control group, whereas KRT17 expression was associated with a reduced number of cells in this growth phase (Fig. 3a). Rates of KRT17-knockdown SMMC-7721 cell apoptosis were also lower than rates in control cells, whereas KRT17 upregulation was associated with increased apoptotic death (Fig. 3b). KRT17 expression was higher on average for patients with a higher T stage in the TCGA LIHC database. As such, we utilized colony formation and CCK-8 assays to examine the relationship between KRT17 and tumor cell proliferation, revealing that KRT17 knockdown markedly impaired SMC-7721 cell proliferation, whereas KRT17 overexpression enhanced the proliferation of HCC-7721 (Fig. 3e-g). Consistent with these findings, subcutaneous tumors generated using SMMC-7721/shKRT17 cells grew more slowly in nude mice than did control cells (Fig. 3h).

\subsection{Assessment of the biological function of KRT17 co- regulated genes in $\mathrm{HCC}$}

As a means of better understanding the potential regulatory roles of KRT17 in HCC, genes with which it was co-expressed were identified using the STRING database (Fig. 4a). Functional enrichment analyses of 11 co-regulated genes were then conducted with ClueGO, revealing these KRT17 co-regulated genes to

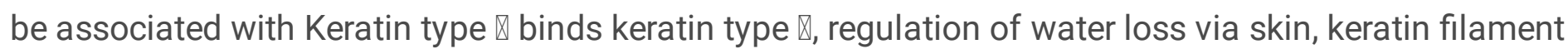
and intermediate filament cytoskeleton organization (Fig. 4b).

\subsection{The relationship between KRT17 expression and HCC tumor immune cell infiltration}


To assess the relationship between KRT17 and the infiltration of immune cells into HCC tumors, we began by comparing immune cell levels in HCC and paracancerous tissue samples (Fig. 5.a). TIMER and sSGSEA analyses revealed that higher KRT17 expression was linked to B cell, CD8 + T cell, CD4 + T cell, macrophage, neutrophil, dendritic cell, central memory CD4 T cell, plasmacytoid dendritic cell, natural killer T cell, myeloid-derived suppressor cell, CD56 natural killer cell, regulatory T cell, and T follicular helper cell infiltration $(P<0.05)$, whereas reduced KRT17 expression was associated with memory $B$ cell and eosinophil infiltration (Fig. 5b-I, P< 0.05). These relationships suggest that KRT17 may serve as a key regulator of $\mathrm{T}$ cell functionality in the context of $\mathrm{HCC}$, underscoring its importance as a regulator of immune infiltration in this oncogenic context.

\section{Discussion}

Keratin proteins including KRT8 and KRT18 serve as important regulators of liver injury, fibrosis, and cancer [25-28]. The prognostic and functional relevance of KRT17 in HCC, however, has not been defined to date. Herein, we analyzed both the TCGA LIHC database and a separate cohort of $44 \mathrm{HCC}$ patients and determined that KRT17 expression levels were significantly higher in HCC tumors relative to normal paracancerous tissues. Elevated KRT17 levels were also identified as a risk factor associated with decrease HCC patient survival.

Through GSEA and functional enrichment analyses, we determined that KRT17 was closely linked to the regulation of many key biological processes including apoptotic signaling, methyl CpG binding, development of the embryonic digestive tract, Notch signaling, and cell cycle pathways. These pathways may be linked to the mechanisms whereby KRT17 governs HCC development and progression.

Dysregulation of the cell cycle and suppression of apoptotic cell death are key hallmarks of oncogenesis, making them prime targets for the treatment of all cancer types [29]. The majority of extant antitumor drugs are anti-mitotic agents, interfering with DNA synthesis and cellular division in a non-targeted manner [30-32]. Herein, we found that the knockdown of KRT17 in HCC cells was associated with G1 phase arrest and a decrease in the frequency of cells in the S phase, while overexpressing KRT17 drove increased rates of apoptotic death. HCC cells. Although there is currently no effective means to inhibit the growth of liver cancer cells, more and more drugs are struggling to target molecular targets for cell growth[33]. Some recent studies proved that growth-inhibiting pathologies (such as E2F and Myc) are effective differentiation inhibitors[34]. We found that knocking down KRT17 was sufficient to suppress the proliferation of HCC cells in vitro and in vivo.

Notch genes encode cell surface receptors that control differentiation and development in myriad species, including humans $[35,36]$. Notch signaling can influence apoptosis, proliferation, pluripotent progenitor cell differentiation, and the formation of cell boundaries. Notch gene mutations can result in profound signaling changes and consequent phenotypic effects [35], and keratin family genes are also linked to many key Notch-induced signaling activities [37, 38]. Herein, our GSEA results revealed KRT17 enrichment 
in the Notch pathway, suggesting that KRT17 may drive dysfunctional HCC cell proliferation, cell cycle progression, and apoptosis at least in part via the Notch signaling pathway.

Tumor immunotherapeutic treatment strategies have revolutionized the standards of care for certain cancer types, and the role of the immune system in the context of cancer progression is increasingly well understood [39,40]. The composition of the tumor microenvironment has also been studied as a prognostic biomarker [41], and there is prior evidence that immune cell infiltration is linked to liver cancer patient survival [42], consistent with our findings. We additionally found that KRT17 expression levels were associated with the degree of immune cell infiltration in HCC patients such that elevated KRT17 expression was positively correlated with macrophage and activated CD4 + T cell infiltration. Together, these data offer detailed insights into the relationship between KRT17 expression and immune markers in LIHC patients. Future work, however, will be required to understand whether KRT17 is a key factor linked with CD $4+\mathrm{T}$ lymphocyte therapy outcomes.

In summary, our results provide multiple lines of evidence confirming that KRT17 is an important regulator of $\mathrm{HCC}$ and a potential prognostic biomarker that can be used to evaluate patients affected by this disease. Specifically, we determine that KRT17 upregulation in HCC tumors is likely to dramatically impact apoptosis, proliferation, and cell cycle progression. In addition, KRT17 may function as a novel immunoregulatory gene in this oncogenic context, underscoring the value of future genomics studies of HCC patient samples.

\section{Declarations}

Acknowledgments: This work was supported by grants from the Social Development Foundation of Nantong City (Grant.nos.JC2019032; MS12019020); Postdoctoral Science Foundation of China (Grant No.2019M661909); Young Medical Talent Project of Jiangsu Province (QNRC2016697).

Authors' contributions: WH and ZXL conceived and designed the experiments. YYC and JC participated in the experiments and drafted the manuscript. ZCT, DHZ, RJ and SJG contributed to the sample collection and interpretation of the data. All authors read and approved the final manuscript.

Funding: none.

Availability of data and materials: The datasets used and/or analyzed during the current study are available from the corresponding author on reasonable request.

Ethics approval and consent to participate: The present study was approved by the Ethics Committee of Nantong University (Nantong, China). Written informed consent was obtained from each participant.

Consent for publication: All persons designated as authors qualify for authorship and have approved the final version of the manuscript.

Competing interests: The authors declare that they have no competing interests. 


\section{References}

1. Bray F, Ferlay J, Soerjomataram I, Siegel RL, Torre LA, Jemal A. Global cancer statistics 2018: GLOBOCAN estimates of incidence and mortality worldwide for 36 cancers in 185 countries. CA Cancer J Clin. 2018;68(6):394-424.

2. Hanouneh IA, Alkhouri N, Singal AG. Hepatocellular carcinoma surveillance in the 21st century: Saving lives or causing harm? Clin Mol Hepatol. 2019;25(3):264-9.

3. Herrmann H, Aebi U. Intermediate filaments: molecular structure, assembly mechanism, and integration into functionally distinct intracellular Scaffolds. Annu Rev Biochem. 2004;73:749-89.

4. Jacob JT, Coulombe PA, Kwan R, Omary MB. Types I and II Keratin Intermediate Filaments. Cold Spring Harb Perspect Biol 2018, 10(4).

5. Jang KH, Yoon HN, Lee J, Yi H, Park SY, Lee SY, Lim Y, Lee HJ, Cho JW, Paik YK, et al. Liver diseaseassociated keratin 8 and 18 mutations modulate keratin acetylation and methylation. FASEB J. 2019;33(8):9030-43.

6. Ku NO, Strnad P, Bantel H, Omary MB. Keratins: Biomarkers and modulators of apoptotic and necrotic cell death in the liver. Hepatology. 2016;64(3):966-76.

7. Wang Z, Yang MQ, Lei L, Fei LR, Zheng YW, Huang WJ, Li ZH, Liu CC, Xu HT. Overexpression of KRT17 promotes proliferation and invasion of non-small cell lung cancer and indicates poor prognosis. Cancer Manag Res. 2019;11:7485-97.

8. Ujiie D, Okayama H, Saito K, Ashizawa M, Thar Min AK, Endo E, Kase K, Yamada L, Kikuchi T, Hanayama $\mathrm{H}$, et al. KRT17 as a prognostic biomarker for stage II colorectal cancer. Carcinogenesis. 2020;41(5):591-9.

9. Li D, Ni XF, Tang H, Zhang J, Zheng C, Lin J, Wang C, Sun L, Chen B. KRT17 Functions as a Tumor Promoter and Regulates Proliferation, Migration and Invasion in Pancreatic Cancer via mTOR/S6k1 Pathway. Cancer Manag Res. 2020;12:2087-95.

10. Li J, Chen Q, Deng Z, Chen X, Liu H, Tao Y, Wang X, Lin S, Liu N. KRT17 confers paclitaxel-induced resistance and migration to cervical cancer cells. Life Sci. 2019;224:255-62.

11. Chivu-Economescu M, Dragu DL, Necula LG, Matei L, Enciu AM, Bleotu C, Diaconu CC. Knockdown of KRT17 by siRNA induces antitumoral effects on gastric cancer cells. Gastric Cancer. 2017;20(6):948-59.

12. Sarlos DP, Yusenko MV, Peterfi L, Szanto A, Kovacs G. Dual role of KRT17: development of papillary renal cell tumor and progression of conventional renal cell carcinoma. J Cancer. 2019;10(21):51249.

13. Yan X, Yang C, Hu W, Chen T, Wang Q, Pan F, Qiu B, Tang B. Knockdown of KRT17 decreases osteosarcoma cell proliferation and the Warburg effect via the AKT/mTOR/HIF1alpha pathway. Oncol Rep. 2020;44(1):103-14.

14. Trierweiler C, Hockenjos B, Zatloukal K, Thimme R, Blum HE, Wagner EF, Hasselblatt P. The transcription factor c-JUN/AP-1 promotes HBV-related liver tumorigenesis in mice. Cell Death Differ. 
2016;23(4):576-82.

15. Xiong H, Hong J, Du W, Lin YW, Ren LL, Wang YC, Su WY, Wang JL, Cui Y, Wang ZH, et al. Roles of STAT3 and ZEB1 proteins in E-cadherin down-regulation and human colorectal cancer epithelialmesenchymal transition. J Biol Chem. 2012;287(8):5819-32.

16. Ashburner M, Ball CA, Blake JA, Botstein D, Butler H, Cherry JM, Davis AP, Dolinski K, Dwight SS, Eppig JT, et al. Gene ontology: tool for the unification of biology. The Gene Ontology Consortium. Nat Genet. 2000;25(1):25-9.

17. Kanehisa M. The KEGG database. Novartis Found Symp. 2002;247:91-101. discussion 101-103, $119-128,244-152$.

18. Franceschini A, Szklarczyk D, Frankild S, Kuhn M, Simonovic M, Roth A, Lin J, Minguez P, Bork P, von Mering C, et al. STRING v9.1: protein-protein interaction networks, with increased coverage and integration. Nucleic Acids Res. 2013;41(Database issue):D808-15.

19. Smoot ME, Ono K, Ruscheinski J, Wang PL, Ideker T. Cytoscape 2.8: new features for data integration and network visualization. Bioinformatics. 2011;27(3):431-2.

20. Bindea G, Mlecnik B, Hackl H, Charoentong P, Tosolini M, Kirilovsky A, Fridman WH, Pages F, Trajanoski Z, Galon J. ClueGO: a Cytoscape plug-in to decipher functionally grouped gene ontology and pathway annotation networks. Bioinformatics. 2009;25(8):1091-3.

21. Bindea G, Galon J, Mlecnik B. CluePedia Cytoscape plugin: pathway insights using integrated experimental and in silico data. Bioinformatics. 2013;29(5):661-3.

22. Li T, Fan J, Wang B, Traugh N, Chen Q, Liu JS, Li B, Liu XS. TIMER: A Web Server for Comprehensive Analysis of Tumor-Infiltrating Immune Cells. Cancer Res. 2017;77(21):e108-10.

23. Hanzelmann S, Castelo R, Guinney J. GSVA: gene set variation analysis for microarray and RNA-seq data. BMC Bioinformatics. 2013;14:7.

24. Lu H, Feng Y, Hu Y, Guo Y, Liu Y, Mao Q, Xue W. Spondin 2 promotes the proliferation, migration and invasion of gastric cancer cells. J Cell Mol Med. 2020;24(1):98-113.

25. Strnad P, Tao GZ, Zhou Q, Harada M, Toivola DM, Brunt EM, Omary MB. Keratin mutation predisposes to mouse liver fibrosis and unmasks differential effects of the carbon tetrachloride and thioacetamide models. Gastroenterology. 2008;134(4):1169-79.

26. Thulin P, Nordahl G, Gry M, Yimer G, Aklillu E, Makonnen E, Aderaye G, Lindquist L, Mattsson CM, Ekblom B, et al. Keratin-18 and microRNA-122 complement alanine aminotransferase as novel safety biomarkers for drug-induced liver injury in two human cohorts. Liver Int. 2014;34(3):367-78.

27. Govaere O, Petz M, Wouters J, Vandewynckel YP, Scott EJ, Topal B, Nevens F, Verslype C, Anstee QM, Van Vlierberghe $\mathrm{H}$, et al. The PDGFRalpha-laminin B1-keratin 19 cascade drives tumor progression at the invasive front of human hepatocellular carcinoma. Oncogene. 2017;36(47):6605-16.

28. Toivola DM, Ku NO, Resurreccion EZ, Nelson DR, Wright TL, Omary MB. Keratin 8 and 18 hyperphosphorylation is a marker of progression of human liver disease. Hepatology. 2004;40(2):459-66. 
29. Evan GI, Vousden KH. Proliferation, cell cycle and apoptosis in cancer. Nature. 2001;411(6835):3428.

30. Evan GI, Wyllie AH, Gilbert CS, Littlewood TD, Land H, Brooks M, Waters CM, Penn LZ, Hancock DC. Induction of apoptosis in fibroblasts by c-myc protein. Cell. 1992;69(1):119-28.

31. Schmitt CA, Lowe SW. Apoptosis and therapy. J Pathol. 1999;187(1):127-37.

32. Evan G, Littlewood T. A matter of life and cell death. Science. 1998;281(5381):1317-22.

33. Pelengaris S, Littlewood T, Khan M, Elia G, Evan G. Reversible activation of c-Myc in skin: induction of a complex neoplastic phenotype by a single oncogenic lesion. Mol Cell. 1999;3(5):565-77.

34. Felsher DW, Bishop JM. Reversible tumorigenesis by MYC in hematopoietic lineages. Mol Cell. 1999;4(2):199-207.

35. Terauchi KJ, Shigeta Y, Iguchi T, Sato T. Role of Notch signaling in granulosa cell proliferation and polyovular follicle induction during folliculogenesis in mouse ovary. Cell Tissue Res. 2016;365(1):197-208.

36. Aster JC, Pear WS, Blacklow SC. The Varied Roles of Notch in Cancer. Annu Rev Pathol. 2017;12:245-75.

37. Arumugam A, Weng Z, Chaudhary SC, Afaq F, Elmets CA, Athar M. Keratin- 6 driven ODC expression to hair follicle keratinocytes enhances stemness and tumorigenesis by negatively regulating Notch. Biochem Biophys Res Commun. 2014;451(3):394-401.

38. Chen Y, Guldiken N, Spurny M, Mohammed HH, Haybaeck J, Pollheimer MJ, Fickert P, Gassler N, Jeon MK, Trautwein C, et al. Loss of keratin 19 favours the development of cholestatic liver disease through decreased ductular reaction. J Pathol. 2015;237(3):343-54.

39. Camidge DR, Doebele RC, Kerr KM. Comparing and contrasting predictive biomarkers for immunotherapy and targeted therapy of NSCLC. Nat Rev Clin Oncol. 2019;16(6):341-55.

40. Carbone DP, Gandara DR, Antonia SJ, Zielinski C, Paz-Ares L. Non-Small-Cell Lung Cancer: Role of the Immune System and Potential for Immunotherapy. J Thorac Oncol. 2015;10(7):974-84.

41. Altorki NK, Markowitz GJ, Gao D, Port JL, Saxena A, Stiles B, McGraw T, Mittal V. The lung microenvironment: an important regulator of tumour growth and metastasis. Nat Rev Cancer. 2019;19(1):9-31.

42. Kurebayashi Y, Ojima H, Tsujikawa H, Kubota N, Maehara J, Abe Y, Kitago M, Shinoda M, Kitagawa Y, Sakamoto M. Landscape of immune microenvironment in hepatocellular carcinoma and its additional impact on histological and molecular classification. Hepatology. 2018;68(3):1025-41.

\section{Figures}



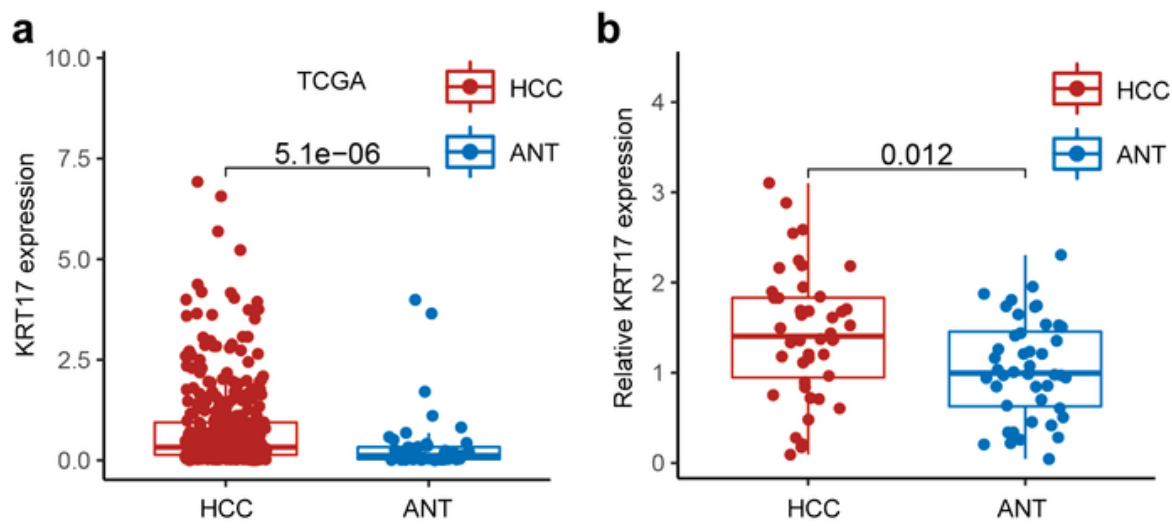

C

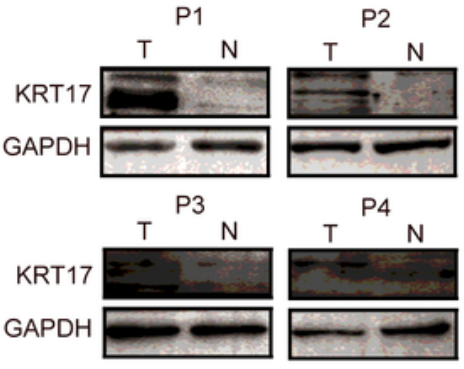

d
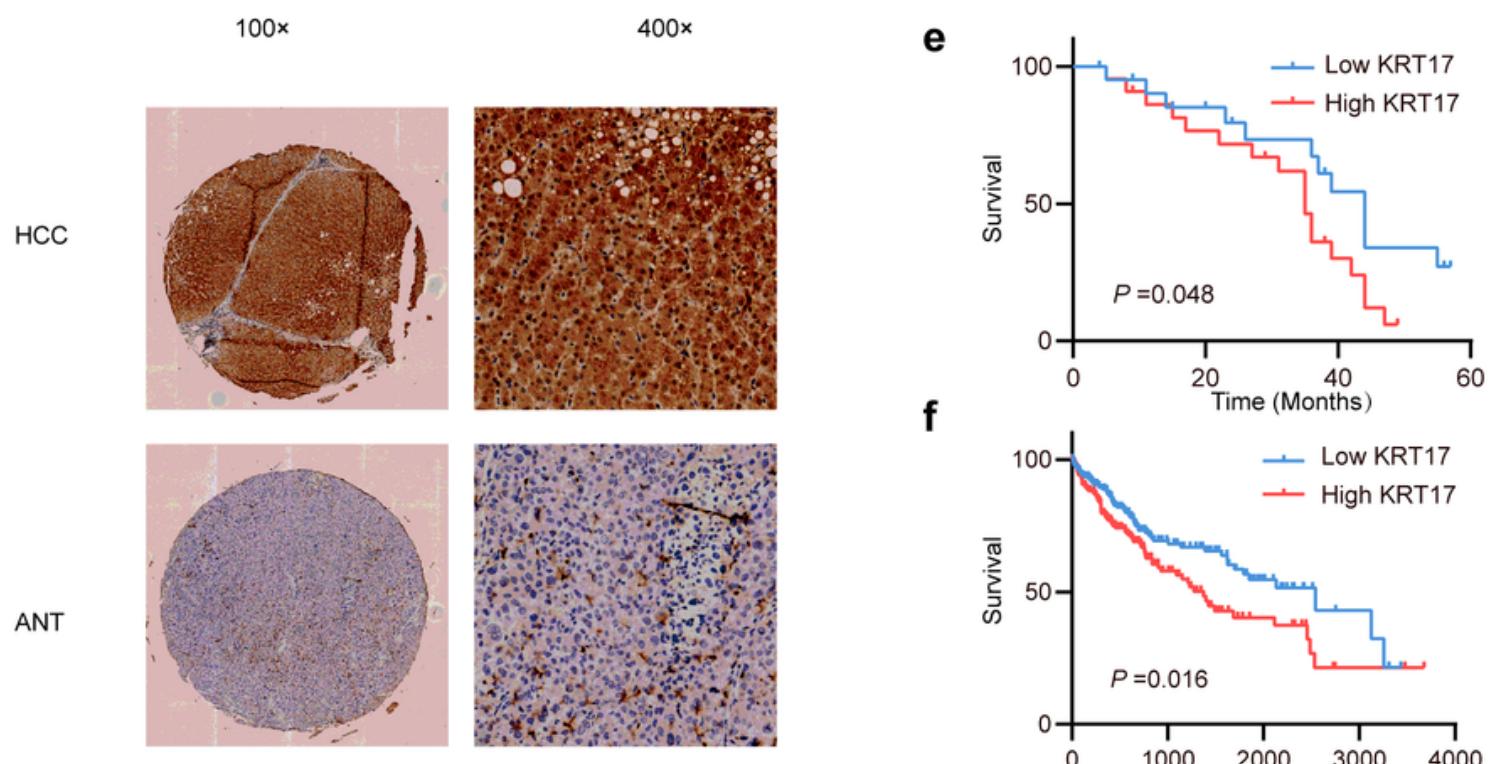

f

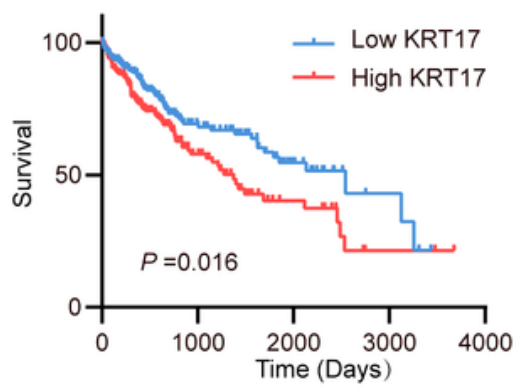

g

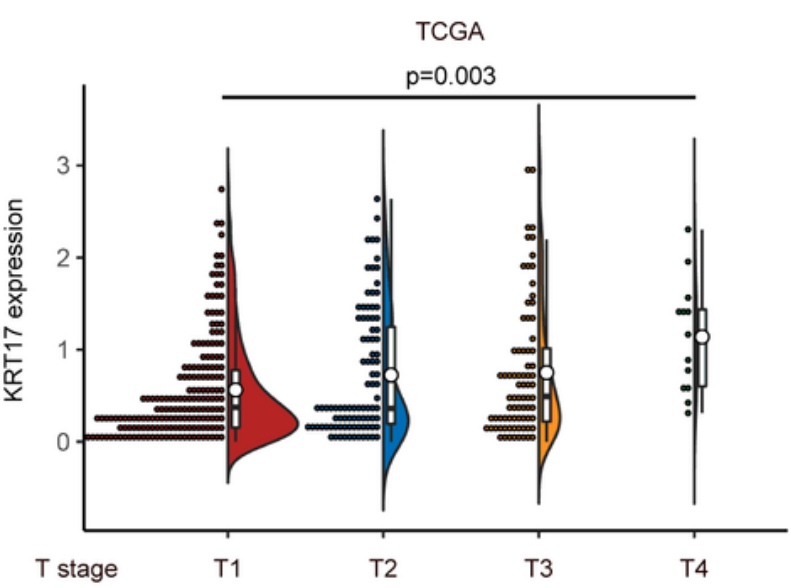

h

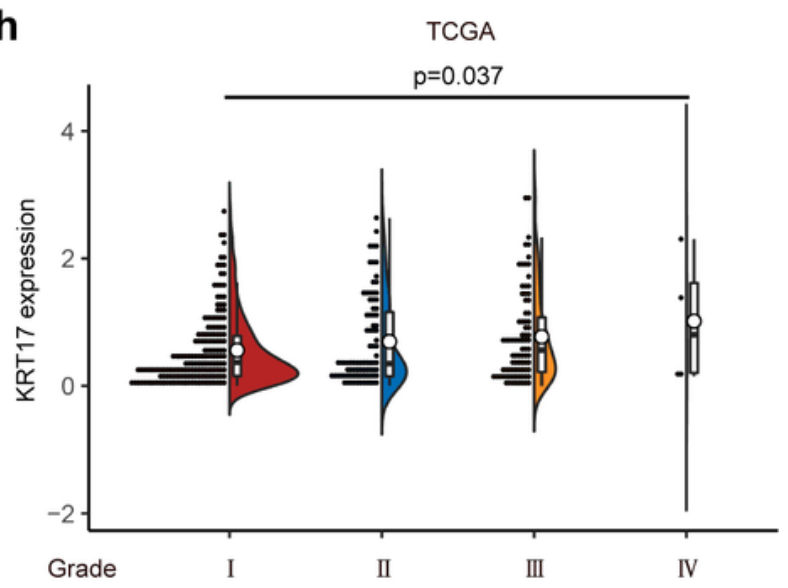

\section{Figure 1}

KRT17 upregulation is evident in HCC tumors and associated with poor prognosis. (a-b) KRT17 expression levels were assessed in HCC tumor and matched paracancerous tissue samples from $44 \mathrm{HCC}$ patients and in samples from the TCGA LIHC database. (c) KRT17 levels in HCC patient tumor and paracancerous tissues were assessed via Western blotting. (d) Representative images of KRT17 staining in HCC and paracancerous tissues are shown. (e-f) The overall survival of HCC with low- and high KRT17 
expression levels was compared via the Kaplan-Meier approach for patients from the TCGA LIHC database and for the 44 patients analyzed in the present study. (g-h) Violin plots were used to assess T and $\mathrm{N}$ stages for patients from the TCGA LIHC database. $\mathrm{P}<0.05$ was considered statistically significant.

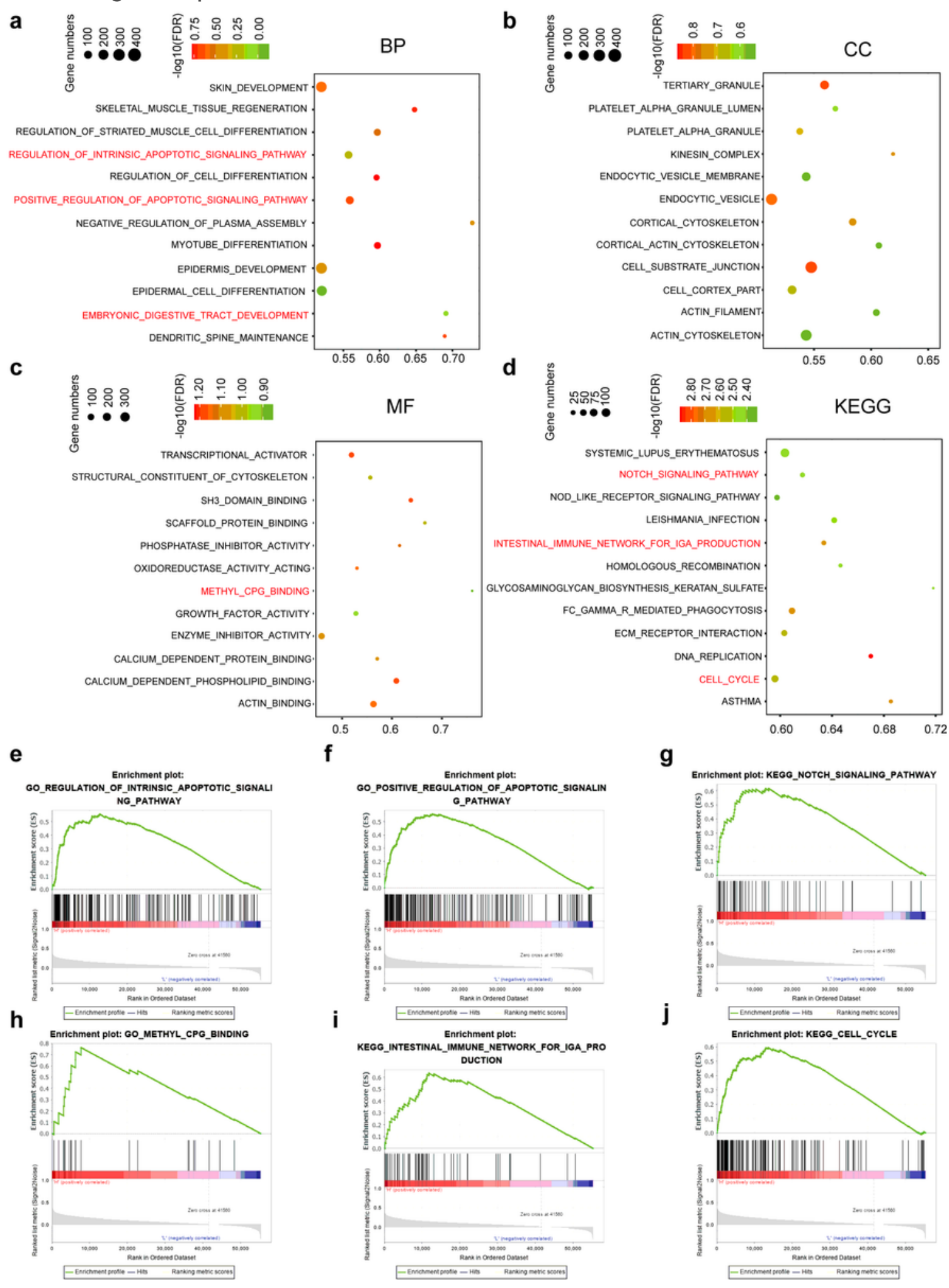

Figure 2

Gene set enrichment analysis-mediated identification of genes co-regulated with KRT17. (a-j) GO and KEGG enrichment analyses were utilized to identify key GSEA-related signaling pathways. 
a

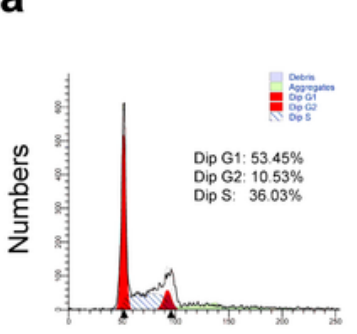

C

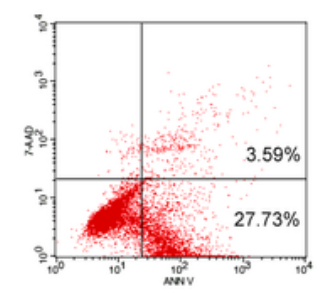

e

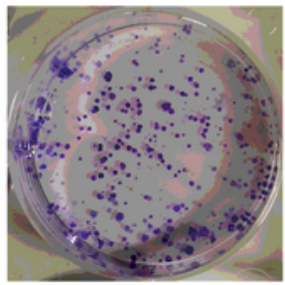

LM3/control

h
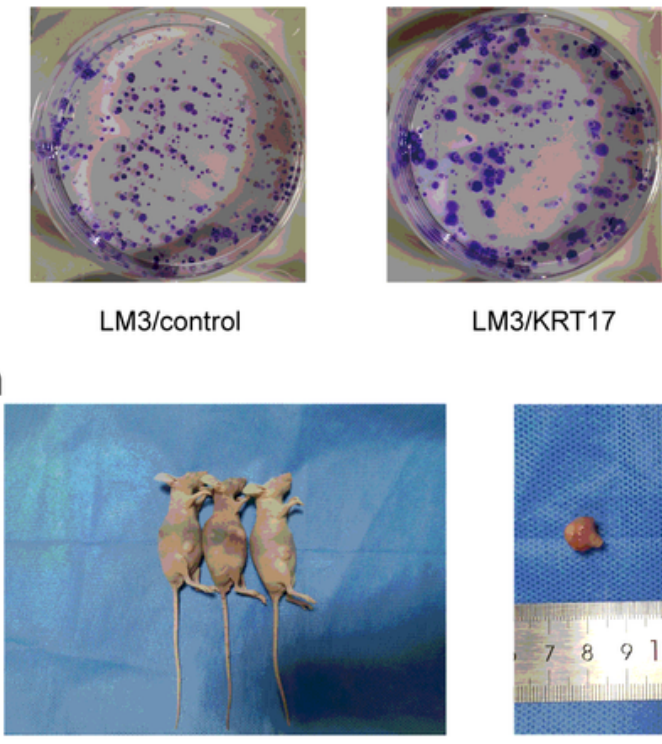

LM3/KRT17

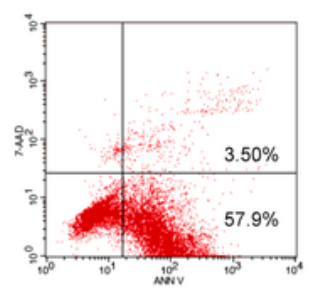

LM3/KRT17
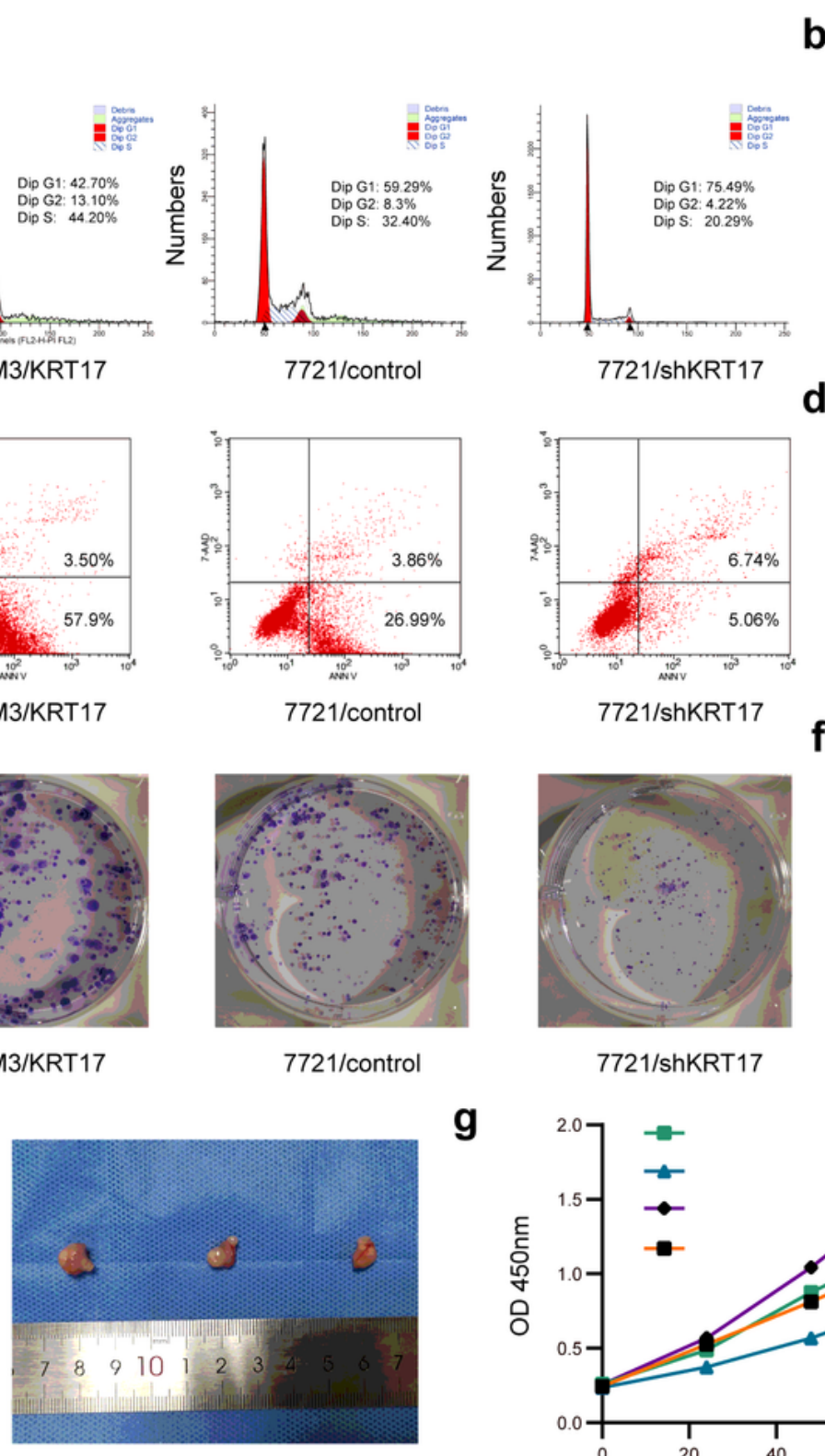

$7721 /$ control

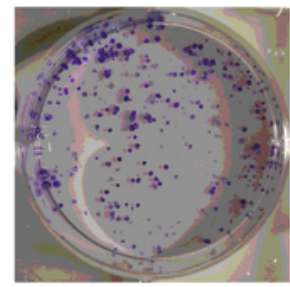

$7721 /$ contro

g
7721/shKRT17

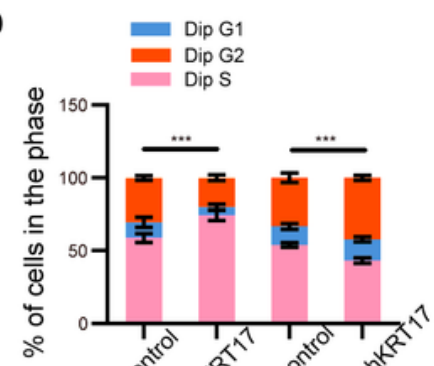

d

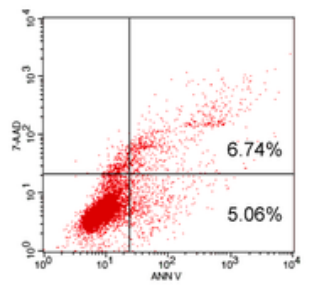

7721/shKRT17

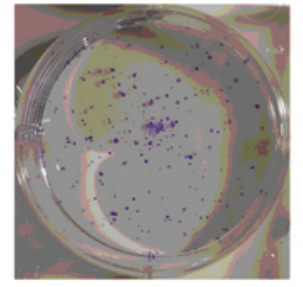

7721/shKRT17
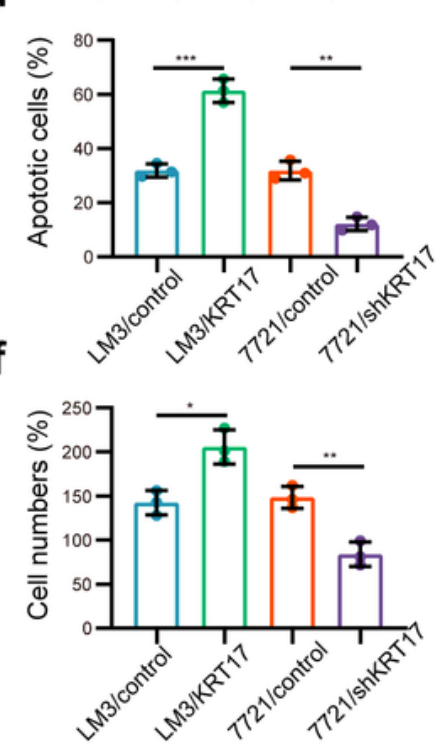
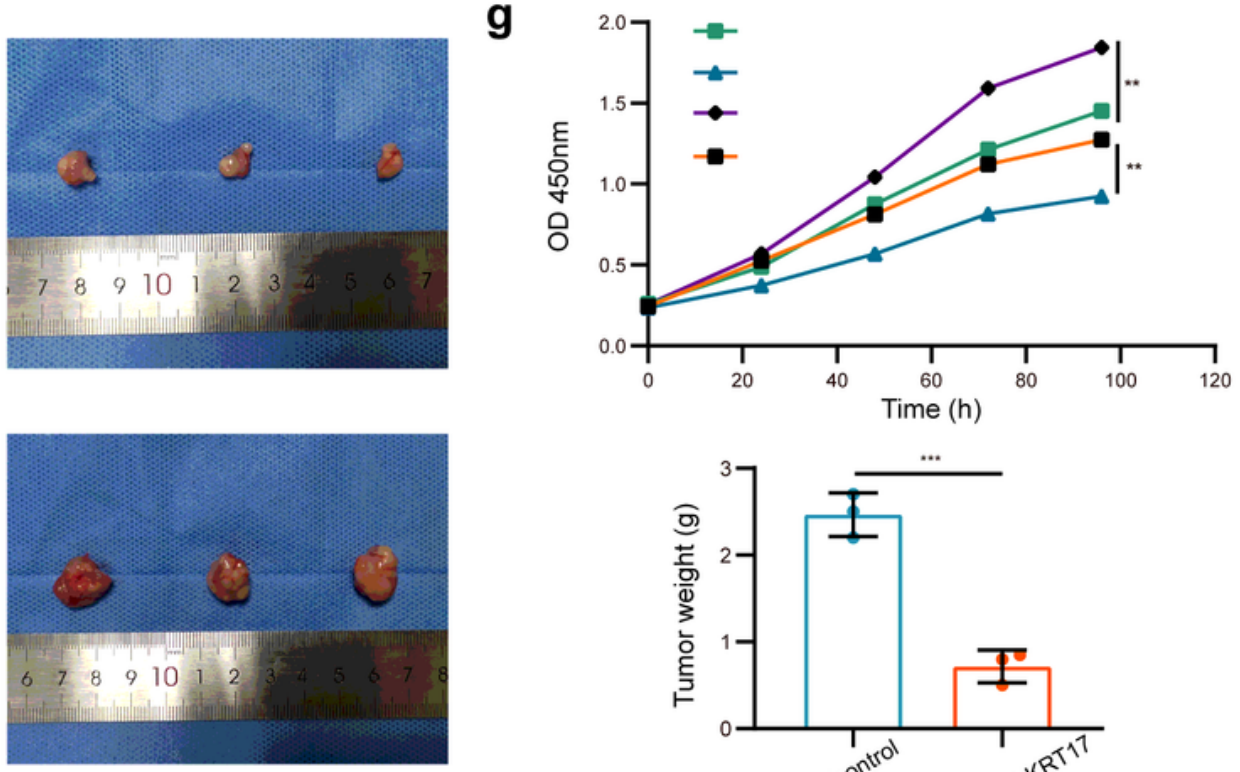

\section{Figure 3}

KRT17 downregulation is linked with HCC cell G1 phase arrest, decreased proliferation, and apoptotic cell death. (a-b) HCC cell cycle progression and apoptosis were analyzed via flow cytometry. (c-e) CCK-8, colony formation, and in vivo analyses were used to assess the relationship between KRT17 and tumor proliferation. ${ }^{*} \mathrm{P}<0.05,{ }^{*} \mathrm{P}<0.05,{ }^{*} * \mathrm{P}<0.001$. 
a

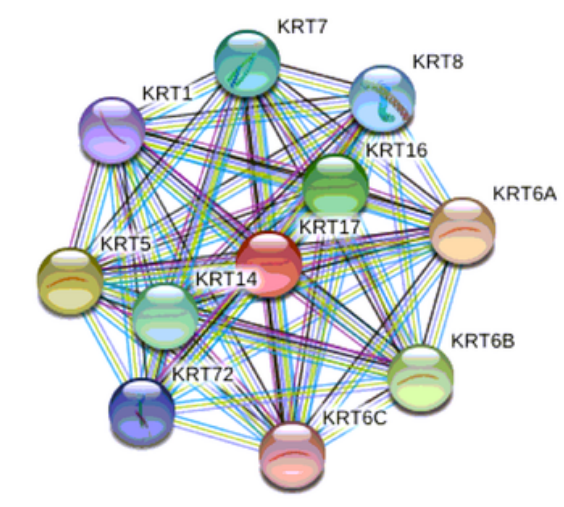

Known Interactions $\quad$ Others Predicted Interactions

$\Theta-\Theta$ from curated databases $\Theta-\Theta$ textmining $\Theta-\Theta$ gene neighborthoo

$\Theta-\ominus$ experimentally defermined $\Theta-\Theta$ coexpression $\Theta-\Theta$ gene fusions

$\Theta-\Theta$ protein homology $\Theta-\Theta$ gene co-occurrence

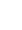

b

으응

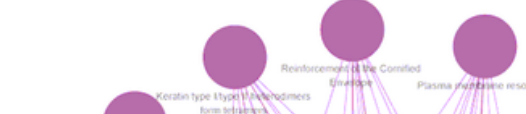

.

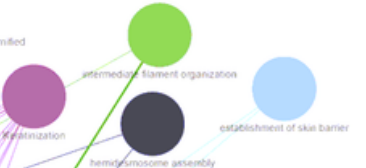
O
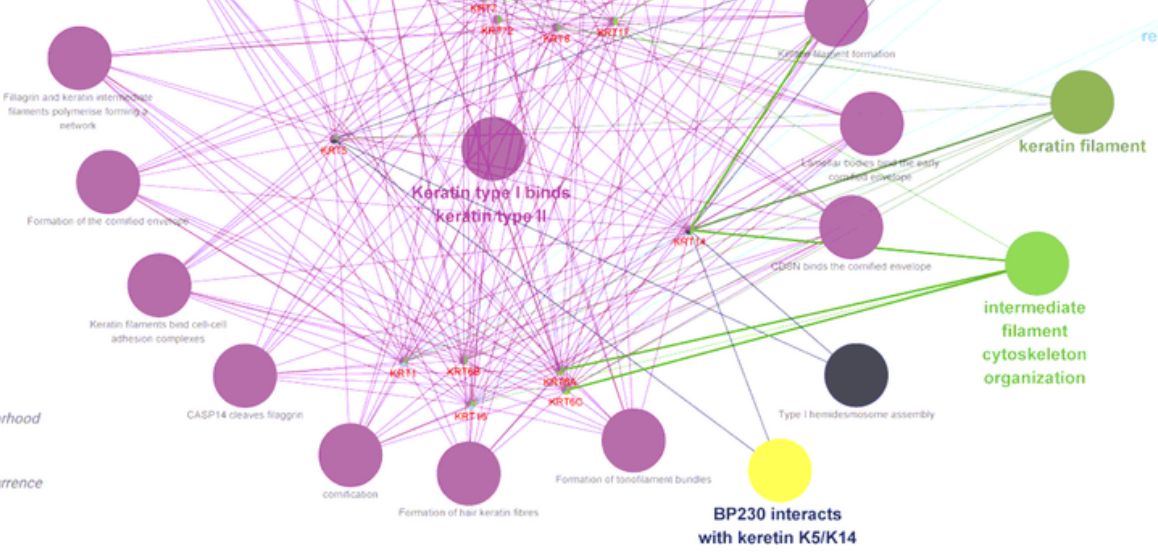

Figure 4

Analysis of KRT17-associated proteins. (a) In total, $11 \mathrm{KRT} 17-$ related proteins were detected via STRING. (b) GO and KEGG analyses were used to identify key signaling pathways associated with these coregulated proteins and visualized with cytoscape. 

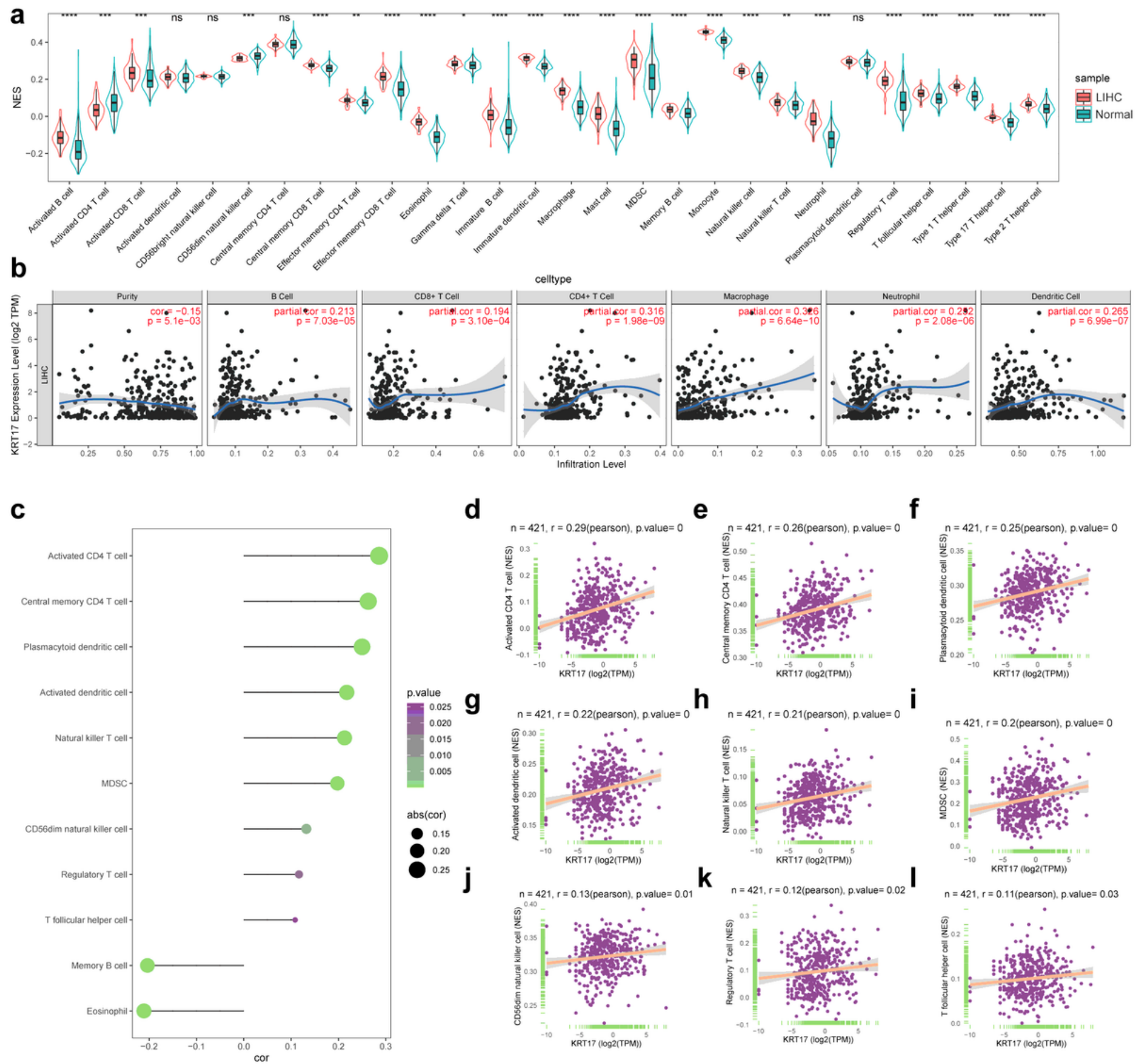

Figure 5

KRT17 expression is correlated with levels of immune infiltration in HCC patients. (a) (b-l) KRT17 expression levels were compared between HCC patients with high and low immune scores as defined through ssGSEA and TIMER analyses. ${ }^{*} P<0.05,{ }^{* \star P} P<0.05$, ${ }^{\star \star \star} P<0.001,{ }^{\star \star \star \star *} P<0.0001$.

\section{Supplementary Files}

This is a list of supplementary files associated with this preprint. Click to download. 
- supfig1.1.tif

- supplementaryfigurelegend.docx 